\title{
Crustáceos decápodos associados às cordas de cultivo do mexilhão Perna perna (Linnaeus, 1758) (Mollusca, Bivalvia, Mytilidae) na Enseada da Armação do Itapocoroy, Penha - SC
}

\author{
Paola Priscilla Brueckheimer de Macedo ${ }^{1,3}$, Setuko Masunari ${ }^{1} \&$ Ricardo Corbetta $^{2}$ \\ ${ }^{1}$ Laboratório de Ecologia de Crustacea, Universidade Federal do Paraná - UFPR, \\ Centro Politécnico, Bairro Jardim das Américas, CP 19020, CEP 81530-900, Curitiba, PR, Brasil \\ ${ }^{2}$ Laboratório de Ciências Ambientais, CTTMar, Universidade do Vale do Itajaí - UNIVALI, \\ Rua Uruguai, 458, CP 360, CEP 88302-900, Itajaí, SC, Brasil \\ ${ }^{3}$ Autor para correspondência: Paola Priscilla Brueckheimer de Macedo, \\ e-mail: paolapbmacedo@yahoo.com.br
}

MACEDO, P.P.B., MASUNARI, S. \& CORBETTA, R. Decapod crustaceans associated with farming ropes of the mussel Perna perna (Linnaeus, 1758) (Mollusca, Bivalvia, Mytilidae) from Armacao do Itapocoroy Bay, Penha - SC. Biota Neotrop. 12(2): http://www.biotaneotropica.org.br/v12n2/en/abstract?inventory+ bn01812022012

\begin{abstract}
This study aimed to describe the assemblage of decapod crustaceans associated with the mussel farming ropes located in the Armação do Itapocoroy Bay, in the municipality of Penha, Santa Catarina State, southern Brazil. The following aspects were assessed: species composition and total weight, succession of species and temporal oscillation of abundance, species richness and Shannon's diversity. The growth data (height and weight of the shells) of the biological substrate (mussels) are also presented. During the nine months of the experiment, associated decapods were collected from 15 ropes (three ropes every other month). A total of 501 decapods were collected that belonged to nine families, 15 genera and 21 species. The most dominant family was Porcellanidae, with seven species, followed by Panopeidae (five) and Pilumnidae (three), while the remaining families were represented by one species each. The most abundant species was Pilumnus dasypodus $(\mathrm{n}=244)$ that together with Synalpheus fritzmuelleri, Pachycheles laevidactylus, Pisidia brasiliensis, Menippe nodifrons, Pachycheles monilifer and Petrolisthes galathinus summed up $88 \%$ of decapod. Species richness increased from three to 15 during the experiment and species diversity showed its maximum in the fifth and ninth month, due to increased availability of shelters within biological substrate composed of living mussels. The total weight of decapods represented only $3 \%$ of the total weight of the farming ropes, due to the smallness of them. This indicates that these animals cannot be considered harmful for the mussel farming. The ropes play a role of shelter and as food resource, since most of these decapods spend all their bentic life in this biotope.
\end{abstract}

Keywords: mytiliculture, associated fauna, richness and species diversity.

MACEDO, P.P.B., MASUNARI, S. \& CORBETTA, R. Crustáceos decápodos associados às cordas de cultivo do mexilhão Perna perna (Linnaeus, 1758) (Mollusca, Bivalvia, Mytilidae) na Enseada da Armação do Itapocoroy, Penha - SC. Biota Neotrop. 12(2): http://www.biotaneotropica.org.br/v12n2/pt/abstract?inventor $\mathrm{y}+\mathrm{bn} 01812022012$

Resumo: Este estudo teve por objetivo descrever a assembleia de crustáceos decápodos associados às cordas de cultivo de mexilhões em uma fazenda marinha localizada na Enseada da Armação do Itapocoroy, no Município de Penha, Santa Catarina, sul do Brasil. Os seguintes aspectos foram tratados: composição de espécies e peso total, sucessão de espécies e oscilação temporal da abundância, riqueza de espécies e diversidade (Shannon). O crescimento do mexilhão em (altura da concha e peso dos indivíduos) também, foram apresentados. Os decápodos foram amostrados de 15 cordas amostradas bimensalmente (três cordas a cada dois meses). Um total de 501 decápodos foram capturados, pertencentes a nove famílias, 15 gêneros e 21 espécies. A família dominante foi Porcellanidae, com sete espécies, seguida de Panopeidae (cinco) e de Pilumnidae (três), enquanto as demais foram representadas por uma espécie. A espécie mais abundante foi Pilumnus dasypodus (n = 244) que, junto com Synalpheus fritzmuelleri, Pachycheles laevidactylus, Pisidia brasiliensis, Menippe nodifrons, Pachycheles monilifer e Petrolisthes galathinus, somou $88 \%$ das ocorrências. A riqueza de espécies aumentou de três para 15 durante o experimento e a diversidade de espécies mostrou seu máximo no quinto mês seguido pelo nono, devido ao aumento da disponibilidade de refúgios no interior do substrato biológico composto por mexilhões vivos. O peso total dos decápodos representou apenas 3\% do peso total das cordas de cultivo, devido ao seu tamanho reduzido. Isto indica que estes animais não podem ser considerados prejudiciais para o cultivou mexilhões. As cordas desempenham o papel de fornecer abrigo e fonte de alimento, já que a maioria destes decápodos passa todo o período bentônico do seu ciclo de vida neste biótopo.

Palavras-chave: mitilicultura, fauna associada, riqueza e diversidade das espécies. 


\section{Introdução}

A mitilicultura é uma atividade que tem crescido mundialmente, fato que também vem sendo observado no Brasil nos últimos anos (Marenzi 1999). A sobre-explotação da pesca devido à falta de cuidado no manejo dos estoques naturais de pescado propiciou o sucesso da aquicultura nas últimas décadas (Young et al. 1999). Dessa forma, a mitilicultura, surgiu como uma fonte alternativa de renda para os pescadores artesanais do litoral do Estado de Santa Catarina, onde se difundiu em praticamente todas as enseadas e baías da costa centro-norte deste Estado que, na última década, tornou-se o segundo maior produtor de mexilhões da América Latina. A vasta maioria das fazendas marinhas cultiva o mexilhão Perna perna (Linnaeus, 1758) (Mollusca, Bivalvia, Mytilidae), em função das condições oceanográficas propícias ao desenvolvimento desta espécie, a qual possui boas qualidades para o cultivo em escala comercial como ampla distribuição geográfica, forte resistência às variações ambientais de temperatura e salinidade, grande capacidade adaptativa, alta taxa de crescimento e elevado valor protéico (Ferreira \& Magalhães 2004).

A primeira referência de cultivo de mexilhões Perna perna para o Estado de Santa Catarina data de 1985, na Enseada da Armação do Itapocoroy, Penha (Marenzi 1999), onde, está localizado atualmente um dos maiores parques de cultivo da América Latina. Nessa enseada, a mitilicultura é realizada em estruturas do tipo "long-line" ou espinhel de meia água, compostas por um cabo horizontal de náilon ou poliamida com 100 metros de comprimento, no qual ficam amarradas verticalmente as cordas de cultivo de mexilhões, que são conhecidas popularmente como pencas de mariscos. A mitilicultura é desenvolvida totalmente no ambiente natural, sem a utilização de rações ou de energia exógena para a circulação de água, tampouco a retirada dos resíduos (Kautsky \& Folke 1991). Contudo, esta produção de mexilhões em interação com sua fauna associada e o meio ambiente traz como consequência mudanças tanto nas características do ecossistema como no aspecto da paisagem. Nos locais onde a mitilicultura é conduzida de forma correta, observa-se um aumento na abundância e na diversidade de espécies de organismos marinhos, proporcionando uma inter-relação trófica dinâmica das espécies neste novo ecossistema (Marenzi \& Manzoni 1998). Por outro lado, nos locais de alta concentração de cultivos, observa-se um enriquecimento orgânico das águas de tal intensidade que apenas espécies tolerantes e com ciclo de vida rápido sobrevivem, determinando a sucessão ecológica local (Tsutsumi et al. 1991, Marenzi \& Manzoni 1998), uma ocorrência típica após um distúrbio ambiental (Connell \& Slatyer 1977).

O cultivo resulta na introdução artificial de espécies e variedades para essas novas áreas e, frequentemente, desloca variedades nativas que tem evoluído em relativo isolamento (Tisdell 1991).A quantidade e o tipo de organismos incrustantes ou vágeis associados às cordas pode se tornar um problema para o cultivo de mexilhões. Essas comunidades incrustantes podem causar mortalidade principalmente nas sementes (juvenis), devido à limitação da superfície disponível para a fixação das larvas (Waterstrat et al. 1980). Podem também reduzir a razão de crescimento pela dificuldade na abertura das valvas, impedindo a entrada de água e alimento (Witman \& Suchanek 1984), causar problemas de flutuação ou romper as estruturas de cultivo suspenso devido ao excesso de peso, ocasionando perdas de estruturas e grandes prejuízos.

Dentre os organismos associados às cordas de mexilhões são conhecidos os hidrozoários, briozoários, ascídias, moluscos, crustáceos e outros animais que também passam ali parte importante de suas vidas (Santos 2006). Seed (1976) relatou que um dos principais fatores responsáveis pela mortalidade dos mexilhões é a predação.
Os gastrópodes predadores mais comuns são Stramonita brasiliensis Claremont \& Reid, 2011 (vulgarmente conhecido como "búzio") e Cymatium parthenopeum (Salis, 1793) (também chamado de "caramujo peludo") que são capazes de abrir a concha e consumir a carne desses bivalves (Manzoni \& Lacava 1998). Outros predadores importantes são algumas espécies de estrelas-do-mar (Inglis \& Gust 2003), caranguejos e siris (DeGraaf \& Tyrrell 2004), pássaros (Hamilton \& Nuds 2003) e peixes (Lappalainen et al. 2004). Alguns parasitas também podem ser encontrados, como o poliqueta Polydora sp., que perfura galerias nas conchas e o caranguejo Pinnotheres maculatus, danificando as brânquias (Marques 1998).

Segundo Seed (1976), os mexilhões em cultivo possuem grande importância ecológica pela criação de um novo substrato sólido em disponibilidade para o estabelecimento de várias espécies associadas, pela remoção de material em suspensão presente em grande quantidade na coluna de água e por atuarem como bioindicadores de poluição costeira.

No Brasil, a fauna associada às cordas de cultivo de mexilhões foi tratada por Marenzi \& Branco (2006) na Enseada da Armação do Itapocoroy, Santa Catarina (SC) e por Camargo (2011) no litoral do Estado de São Paulo. Este estudo tem como objetivos listar as espécies de crustáceos decápodos associados às cordas de mexilhões e caracterizar temporalmente as variações de riqueza, abundância e biomassa desta assembléia ocorrente na Enseada da Armação do Itapocoroy, Penha, SC.

\section{Material e Métodos}

O presente estudo foi desenvolvido na Enseada da Armação do Itapocoroy, no município de Penha, Santa Catarina (Figura 1a), na área de cultivo do Centro Experimental de Maricultura da Universidade do Vale do Itajaí - UNIVALI (Figura 1b). Este município está localizado no litoral centro-norte de Santa Catarina, (26 46' 56" S e $48^{\circ} 38^{\prime}$ 42 " O), e possui uma área de $60,3 \mathrm{~km}^{2}$ fazendo divisa com Navegantes (Sul), Balneário Piçarras (Oeste) e com o Oceano Atlântico (Leste e Norte). As estruturas de cultivo utilizadas são denominadas cordas de mexilhões (Figura 1c).

O cultivo foi iniciado a partir de mexilhões juvenis (sementes) de Perna perna, com altura média de concha de cerca de $25 \mathrm{~mm}$ obtidos dos maricultores e de coletores. As sementes foram introduzidas em sacos de redes de algodão com auxílio de um cano de PVC, que, por sua vez foram inseridos dentro de outro saco de rede de náilon. Nesta etapa, o cano de PVC é subtraído. Essa técnica denomina-se "Francês" devido à sua origem (Marenzi \& Branco 2006) e este conjunto de sacos com sementes constitui uma corda. $\mathrm{O}$ saco de rede de algodão decompõe-se rapidamente, porém não antes dos mexilhões juvenis se fixarem uns sobre os outros por meio do crescimento do bisso, formando um longo e firme agregado. Em seguida, os mexilhões transpõem o saco de rede de náilon, assentando-se na face externa do mesmo, onde crescem até atingir o tamanho comercial. No dia 18 de setembro de 2006 foram imersas 41 cordas de cultivo de mexilhões, cada uma medindo 1 metro de comprimento e pesando aproximadamente $1 \mathrm{~kg}$. O experimento teve duração de nove meses e a primeira amostragem foi realizada em novembro, após um mês do início do experimento e as restantes com frequiência bimestral (dezembro, fevereiro, abril e junho de 2006), num total de cinco amostragens. Em cada amostragem, três cordas foram retiradas da água, com auxílio de um guincho da embarcação, totalizando 15 cordas amostradas. Após o período de nove meses o peso em demasia das 26 cordas de mexilhões restantes, causou o rompimento da estrutura de cultivo "long-line" inviabilizando a continuidade do estudo. Nesse período, ocorreram colonizações de outros organismos por meio de migração e/ou de transporte de ovos e larvas. 


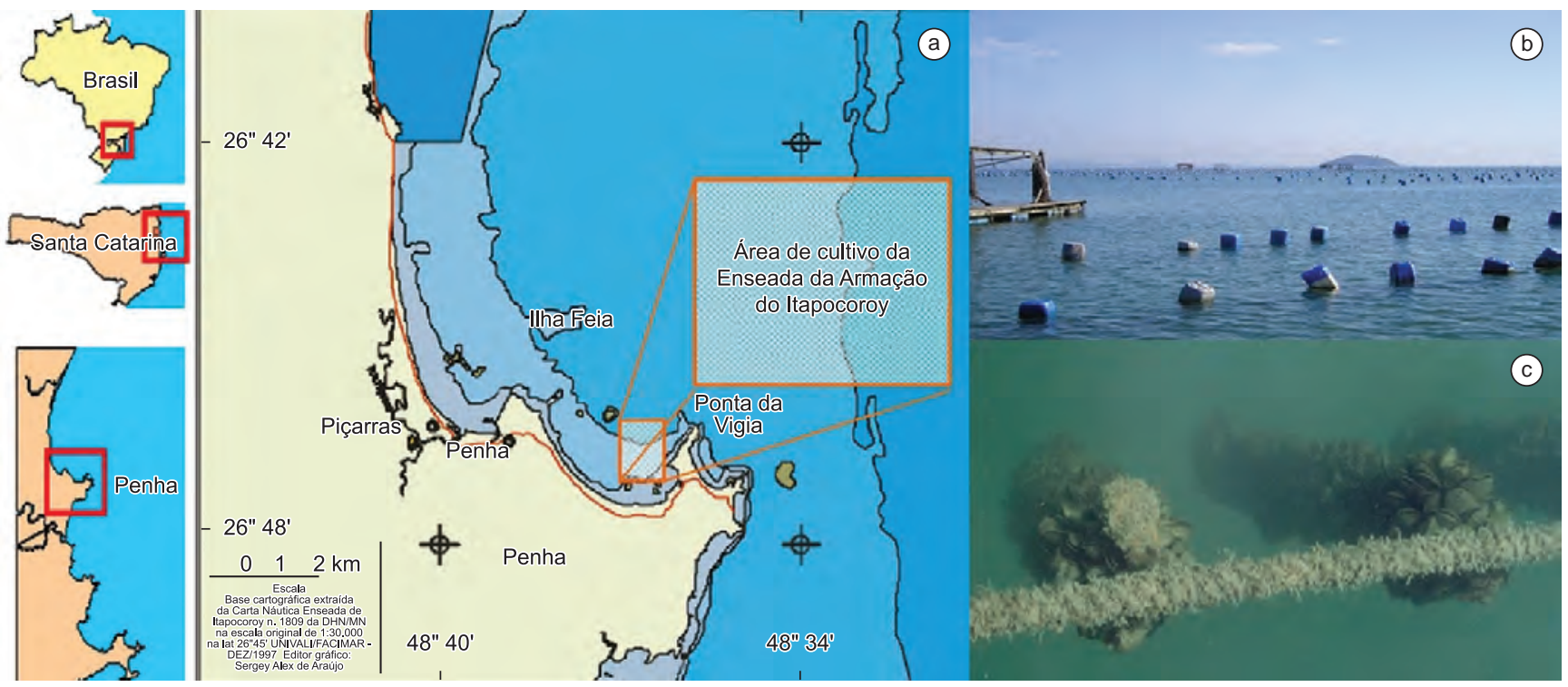

Figura 1. (a) Mapa do litoral do Município de Penha, SC (Fonte: Laboratório de Geoprocessamento Costeiro da UNIVALI) com a indicação da área de cultivo de mexilhões na Enseada da Armação do Itapocoroy; (b) Vista parcial da área de cultivo dentro da enseada e (c) Duas cordas de cultivo submersas e ainda amarradas ao long-line.

Figure 1. (a) Map of the coast of municipality of Penha, SC (Source: GIS Laboratory of Coastal UNIVALI) with the indication of the mussel farming area at Armação do Itapocoroy Bay; (b) Parcial landscape of farming area inside the bay and (c) two submerged cultivation ropes tied to the long-line.

No barco, cada uma das três cordas de cultivo de mexilhões foi acomodada em uma caixa plástica distinta para que os organismos de uma corda não se misturassem com os das outras cordas. Em terra, as cordas foram embaladas em sacos plásticos, transportadas para o Laboratório de Ciências Ambientais na UNIVALI de Itajaí e armazenadas em freezer até seu processamento. De cada amostragem, um lote de 60 mexilhões foi separado aleatoriamente, os quais foram pesados com auxílio de uma balança de precisão e a altura das conchas medidas com paquímetro digital para verificar o aumento de biomassa e tamanho dos mexilhões. As triagens dos crustáceos decápodos foram realizadas após o descongelamento das amostras; estes foram identificados, contados e pesados com os equipamentos acima citados, fixados em formol $4 \%$ e conservados em álcool $70 \%$. A classificação dos decápodos do presente estudo seguiu a proposta de Melo (1996, 1999), Ng et al. (2008) e De Grave et al. (2009). Os crustáceos decápodos do presente estudo estão depositados na Coleção Crustacea, no Laboratório de Invertebrados do Museu de História Natural Capão da Imbuia (MHNCI) sob os lotes: MHNCI 2866; MHNCI 2851; MHNCI 2853 - 2857; MHNCI 2848 - 2849; MHNCI 2850; MHNCI 2859 - 2865; MHNCI 2858 e MHNCI 2852.

\section{Análise dos Dados}

A estrutura da assembleia de decápodos associados às cordas de cultivo de mexilhões foi examinada com base em dois aspectos importantes da sua organização: o número de espécies e as respectivas abundâncias relativas (Giller 1984). A partir desses dados foram calculados os índices biológicos de riqueza e diversidade de Shannon (H') $(\log$ e), na tentativa de resumir as informações e facilitar futuramente a comparação entre este habitat e outros naturais ou artificiais. Foram utilizadas as médias de abundância total e relativa de cada espécie ao longo do tempo de cultivo. As análises foram realizadas através do programa PRIMER 6. Foi utilizada a análise de variância unifatorial (ANOVA) para comparar as médias da altura e peso dos mexilhões cultivados. No caso de existência de diferença significativa $(\mathrm{p}=0,05)$, realizou-se o teste "a posteriori" de Tukey
HSD para saber qual média apresentou a diferença entre os meses. As análises foram realizadas utilizando o programa computacional $\mathrm{R}$.

\section{Resultados}

\section{O substrato biológico Perna perna:}

Os mexilhões cultivados atingiram uma altura média da concha de 70,07 $\pm 25,26 \mathrm{~mm}$ no final de nove meses. Como as sementes (juvenis) mediram $25 \pm 0,25 \mathrm{~mm}$; isto equivale a um incremento médio mensal de 4,74 $\mathrm{mm}$. Houve, entretanto, maior velocidade de crescimento das conchas entre o mês um ao três e do quinto ao nono (Figura 2). Entre o terceiro e quinto mês de amostragem não houve diferença significativa em relação à altura $(\mathrm{F}=285,58$; $\mathrm{p}<2,2 \mathrm{e}-16)$. O peso médio de cada mexilhão ao final do estudo foi de $25,25 \pm 11,59 \mathrm{~g}$, resultado de um incremento médio mensal de $1,6 \mathrm{~g}$; entre os meses três e cinco não houve diferença significativa no peso $(\mathrm{F}=158,68 ; \mathrm{p}=2$,2e-16) ocorrendo um declínio no peso médio total, porém no restante do período de cultivo, não houve diferença significativa entre os meses, havendo sucessivos incrementos de peso (Figura 2). As cordas que pesavam aproximadamente $1 \mathrm{~kg}$ no início do cultivo atingiram o peso médio de $6,220 \mathrm{~kg}$ cada uma, num incremento médio mensal de aproximadamente $580 \mathrm{~g}$ por corda em nove meses de cultivo.

\section{A fauna de decápodos associados às cordas de cultivo de mexilhões:}

Um total de 501 exemplares foi registrado, sendo representados por 21 espécies de decápodos pertencentes a 15 gêneros e nove famílias (Tabela 1). Destas, 12 foram braquiúros, sete anomuros e uma de cada camarão carídeo e peneídeo.

A riqueza da assembléia aumentou de três a 15 espécies e a diversidade de espécies, de $0,36 \pm(0,36)$ a $1,31 \pm(0,15)$ ao longo dos meses de cultivo. Por outro lado, a abundância aumentou de três 
até 262 indivíduos no sétimo mês e diminuiu para 164 no último mês (Tabela 2).

As seguintes espécies perfizeram $88 \%$ de abundância total: Pilumnus dasypodus, Synalpheus cf. fritzmuelleri, Pachycheles laevidactylus, Pisidia brasiliensis, Menippe nodifrons, Pachycheles monilifer e Petrolisthes galathinus. As demais espécies (14) representaram em conjunto apenas $12 \%$ da abundância total (Tabela 3).

Nenhuma espécie foi de ocorrência constante durante todo o período de estudo. A mais frequente e abundante foi Pilumnus dasypodus, que ocorreu em quatro dos cinco meses amostrados, totalizando 244 indivíduos. Pachycheles laevidactylus,

Tabela 1. Lista das espécies dos decápodos associados às cordas de cultivo de mexilhões na Enseada da Armação do Itapocoroy.

Table 1. List of species of decapods associated with strings of growing mussels in the Bay of Armacao Itapocoroy.

\section{Filo Arthropoda Latreille, 1829}

Classe Malacostraca Latreille, 1802

Ordem Decapoda Latreille, 1802

Subordem Dendrobranchiata Bate, 1802

Infraordem Penaeidea (Calazans, 1993)

Família Solenoceridae Wood-Mason \& Alcock, 1891

Pleoticus muelleri (Bate, 1888)

Subordem Pleocyemata Burkenroad, 1963

Infraordem Anomura MacLeay, 1838

Família Porcellanidae Haworth, 1825

Pachycheles laevidactylus Ortmann, 1892

Pachycheles monilifer (Dana, 1852)

Petrolisthes armatus (Gibbes, 1850)

Petrolisthes galathinus (Boch, 1802)

Pisidia brasiliensis Haig in Rodrigues da Costa, 1968

Porcellana platycheles (Pennant, 1777)

Porcellana sayana (Leach, 1820)

Infraordem Brachyura Linnaeus, 1758

Família Pilumnidae Samouelle, 1819

Pilumnoides coelhoi Guinot \& Macpherson, 1987

Pilumnus dasypodus Kingsley, 1879

Pilumnus reticulatus Stimpson, 1860

Família Panopeidae Ortmann, 1893

Acantholobulus schmitti (Rathbun, 1930)

Panopeus rugosus A. Milne Edwards, 1880

Panopeus lacustris Desbonne, 1867

Panopeus austrobesus Williams, 1983

Hexapanopeus paulensis Rathbun, 1930

Família Menippidae Ortmann, 1893

Menippe nodifrons Stimpson, 1859

Família Portunidae Rafinesque, 1815

Callinectes danae (Smith, 1869)

Família Epialtidae MacLeay, 1838

Libinia spinosa H. Milne Edwards, 1834

Família Grapsidae MacLeay, 1838

Pachygrapsus transversus Gibbes, 1850

Infraordem Caridea Dana, 1852

Família Alpheidae Rafinesque, 1815

Synalpheus frizmuelleri Coutière, 1901
Psidia brasiliensis, Pachycheles monilifer, Petrolisthes galathinus ocorreram em três dos cinco meses amostrados, com abundância de 43, 33, 18 e 16 indivíduos, respectivamente. Pilumnus reticulatus apesar da baixa abundância (8) nas amostragens, também ocorreu em três dos cinco meses de amostragens, de modo descontínuo. Pachygrapsus transversus, Petrolisthes armatus, Menippe nodifrons e Hexapanopeus paulensis ocorreram em duas das cinco amostragens. Synalpheus cf. fritzmuelleri foi a segunda espécie mais abundante (62 indivíduos), apesar de sua ocorrência restrita às duas últimas amostragens. Porcellana platycheles, Panopeus lacustris e P. rugosus foram registradas apenas uma vez, mas cada uma dessas espécies representou mais de $1 \%$ da amostragem. As demais espécies tiveram ocorrência rara, ou seja, baixa freqüência e abundância, representando cada espécie menos de $1 \%$ do total de decápodos: Panopeus austrobesus, Porcellana sayana, Pilumnoides coelhoi, Callinectes danae, Pleoticus mueller, Libinia spinosa e Acantholobulus schmitti (antigo Hexapanopeus schmitti) (Tabela 3)

A biomassa média do conjunto das espécies de decápodos associados variou de $0,2 \mathrm{~g}$ (mês 1) a 38,77 g (mês 7); o peso médio dos mesmos ao longo dos meses foi crescente até o sétimo mês $(38,77 \mathrm{~g})$, e houve uma leve diminuição no nono mês $(22,16 \mathrm{~g}$ ) (Tabela 2). A biomassa total de decápodos nos nove meses de amostragem foi de apenas 207,3 g. As cinco espécies que mostraram maior contribuição na biomassa total foram: Pilumnus dasypodus, Menippe nodifrons, Pachycheles laevidactylus, Synalpheus cf. fritzmuelleri e Pisidia brasiliensis. Embora Menippe nodifrons tenha sido representado por somente 24 indivíduos aparentemente juvenis, estes foram os de maior contribuição na biomasssa total, em função do maior porte atingido pela espécie. Por outro lado, Pilumnus dasypodus apresentou maior biomassa total $(123,28 \mathrm{~g})$, com a contribuição de 183 indivíduos adultos no mês sete e 45 no mês nove.

\section{Discussão}

O crescimento médio dos mexilhões do presente estudo $(70,07 \pm 25,26 \mathrm{~mm}$ em altura da concha em nove meses) apresentou-se inferior ao encontrado por Marenzi \& Branco (2006) que, em oito meses de cultivo obtiveram uma altura de concha média de $70,7 \mathrm{~mm}$. Isto pode ser explicado devido à diferença no tamanho das sementes. Marenzi \& Branco (2006) iniciaram seu estudo com sementes de

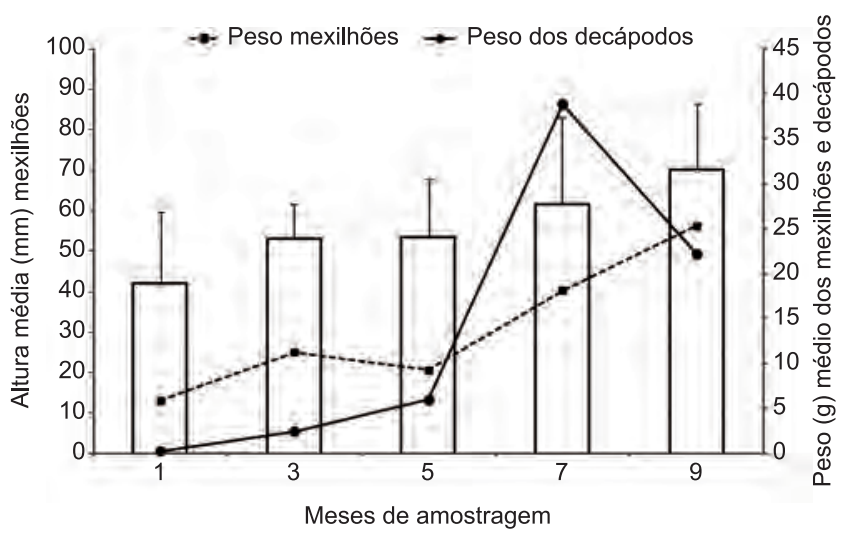

Figura 2. Cordas de cultivo de mexilhão Perna perna. Altura média ( $\mathrm{mm})$ e peso médio (g) individual das conchas dos mexilhões (média de 180 mexilhões por mês de amostragem) e peso médio total dos decápodos associados às cordas (média das três cordas), ao longo do experimento.

Figure 2. Rope cultivation of mussels Perna perna. The average height $(\mathrm{mm})$ and weight $(\mathrm{g})$ of individual shells of mussels (average of 180 mussels per month of sampling) and total weight of decapods associated with ropes (average of three ropes), along the experiment. 
Decápodos associados às cordas de cultivo do Mexilhão Perna perna, Penha - SC

Tabela 2. Decápodos associados às cordas de cultivo de mexilhões na Enseada da Armação do Itapocoroy. Abundância, riqueza, riqueza acumulada, biomassa e diversidade de Shannon das espécies ao longo do experimento.

Table 2. Decapods associated with ropes of mussel cultivated in the Bay of Armação of Itapocoroy. Abundance, specie richness, accumulatet specie richness, biomass and Shannon diversity of species throughout the experiment.

\begin{tabular}{|c|c|c|c|c|c|c|}
\hline Meses & 1 & 3 & 5 & 7 & 9 & $\sum$ \\
\hline espécies & \multicolumn{6}{|c|}{ Abundância das espécies nos meses } \\
\hline Pilumnus dasypodus & 0 & 3 & 13 & 183 & 45 & 244 \\
\hline Synalpheus fritzmuelleri & 0 & 0 & 0 & 21 & 41 & 62 \\
\hline Pachycheles laevidactylus & 0 & 3 & 3 & 37 & 0 & 43 \\
\hline Psidia brasiliensis & 0 & 0 & 21 & 10 & 2 & 33 \\
\hline Menippe nodifrons & 0 & 0 & 0 & 2 & 22 & 24 \\
\hline Pachycheles monilifer & 0 & 0 & 6 & 2 & 10 & 18 \\
\hline Petrolisthes galathinus & 0 & 0 & 12 & 3 & 1 & 16 \\
\hline Porcellana platycheles & 0 & 0 & 0 & 0 & 9 & 9 \\
\hline Hexapanopeus paulensis & 0 & 0 & 0 & 1 & 8 & 9 \\
\hline Pachygrapsus tranversus & 1 & 8 & 0 & 0 & 0 & 9 \\
\hline Panopeus lacustres & 0 & 0 & 0 & 0 & 8 & 8 \\
\hline Pilumnus reticulatus & 0 & 1 & 0 & 2 & 5 & 8 \\
\hline Panopeus rugosus & 0 & 0 & 0 & 0 & 5 & 5 \\
\hline Panopeus austrobesus & 0 & 0 & 0 & 0 & 3 & 3 \\
\hline Petrolisthes armatus & 1 & 1 & 0 & 0 & 0 & 2 \\
\hline Porcellana sayana & 0 & 0 & 0 & 0 & 2 & 2 \\
\hline Pilumnoides coelhoi & 0 & 0 & 0 & 0 & 2 & 2 \\
\hline Libinia spinosa & 0 & 0 & 0 & 1 & 0 & 1 \\
\hline Callinectes danae & 1 & 0 & 0 & 0 & 0 & 1 \\
\hline Acantholobulus schmitti & 0 & 0 & 0 & 0 & 1 & 1 \\
\hline Pleoticus muelleri & 0 & 0 & 0 & 1 & 0 & 1 \\
\hline Abundância & 3 & 16 & 55 & 262 & 164 & 501 \\
\hline Riqueza de espécies & 3 & 5 & 5 & 11 & 15 & \\
\hline Riqueza acumulada & 3 & 6 & 9 & 14 & 21 & \\
\hline Peso (média) total dos decápodes & 0,2 & 2,04 & 5,93 & 38,77 & 22,16 & \\
\hline Diversidade (Shannon_H) & $0,36( \pm 0,36)$ & $0,96( \pm 0,22)$ & $1,37( \pm 0,02)$ & $0,58( \pm 0,49)$ & $1,31( \pm 0,15)$ & \\
\hline
\end{tabular}

$30,0 \mathrm{~mm}$ em média, um valor relativamente maior do que as sementes usadas neste experimento (25,0 $\mathrm{mm}$ em média). Henriques et al. (2004) recomenda iniciar um cultivo com sementes superiores a 40,0 mm para atingir tamanho comercial em menos tempo. Entretanto, devido à dificuldade na obtenção das sementes nem sempre é possível manter esse tamanho mínimo sugerido.

Outro fator que pode ter influenciado essa diferença no crescimento foi o aumento desordenado das áreas de cultivo na região. Segundo Suplicy (2000), o conceito de capacidade de suporte de um ecossistema é derivado de um estudo da ecologia de populações. Outros autores consideram ainda a capacidade suporte como sendo o ponto onde a biomassa de uma determinada população para de crescer, e o montante de biomassa mantida nestas condições é considerado como capacidade de suporte máxima. Além disso, o referido autor afirma que, em casos de elevadas densidades de cordas de cultivo, estas funcionam como "paredes submersas", retendo e causando uma sedimentação excessiva de materiais em suspensão na água. Se as estruturas de cultivo estiverem localizadas em áreas de pequena profundidade e de circulação restrita, os biodepósitos (fezes e pseudofezes) e animais que caem do cultivo causam impactos negativos no sedimento podendo provocar eutrofização do biótopo prejudicando o cultivo de forma geral.

Cultivos densos como na Enseada do Brito (Palhoça, Santa Catarina), onde os mexilhões atingiam o tamanho comercial de
$80,0 \mathrm{~mm}$ em seis a sete meses, atualmente somente o atingem em 12 a 13 meses Suplicy (2000).

Entre o primeiro e o terceiro mês de cultivo, houve grande crescimento dos mexilhões. Isto pode estar relacionado com o período de amostragem (verão), período no qual a enseada é fortemente influenciada pelo aporte de águas continentais, principalmente do Rio Itajaí-Açu o qual é incrementado pelas precipitações que ocorreram nas áreas continentais, além daquela precipitação ocorrida no próprio local de cultivo (Schettini et al. 1999, Carvalho et al. 1999). Esse aporte traz como consequiência todas as substâncias lançadas neste rio: nutrientes inorgânicos e orgânicos, metais traço e compostos orgânicos persistentes (Schettini et al. 1997). Essa maior quantidade de nutrientes, nesse período, promove alimento para esses bivalves, explicando esse maior crescimento nesse período, em contra partida, esse aporte é caracterizado por uma salinidade extremamente baixa (Schettini et al. 1999), aliado aos maiores valores de temperaturas da água, (de $28^{\circ} \mathrm{C}$ a $30^{\circ} \mathrm{C}$ ) que ocorrem nos meses de janeiro e fevereiro nessa enseada (Marenzi \& Branco 2006) e, segundo Loo \& Rosenberg (1983), essa alta temperatura diminui a capacidade de filtração dos mexilhões, influenciando negativamente o seu crescimento nesse período de temperaturas elevadas promovendo estresse para esses organismos. Esse estresse gerado pelo aumento da temperatura provoca ainda a desova dos organismos, que no caso da espécie do mexilhão Perna perna pode apresentar desovas ao longo de todo ano, 
Tabela 3. Decápodos associados às cordas de mexilhões cultivados na Enseada da Armação do Itapocoroy. Ocorrência das espécies (barras horizontais) e oscilação da abundância dos decápodos (número acima das barras horizontais) durante o experimento.

Table 3. Decapods associated with ropes of mussel cultivated in the Bay of Armação of Itapocoroy. Occurrence of species (horizontal bars) and oscillation of the abundance of decapods (number above the horizontal bars) during the experiment.

\begin{tabular}{lcccccc}
\hline \multirow{2}{*}{ Espécies } & Meses & 1 & 3 & 5 & 7 & 9 \\
\cline { 2 - 6 } & Data & $18 / 10 / 06$ & $12 / 12 / 06$ & $21 / 02 / 07$ & $06 / 04 / 07$ & $14 / 06 / 07$ \\
\hline
\end{tabular}

1 Pilumnus dasypodus

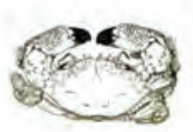

3

13

183

45

2 Pachycheles laevidactylus

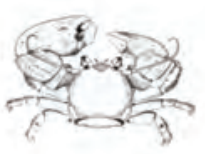

3

3

37

3 Pisidia brasiliensis

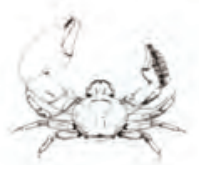

21

10

2

4 Pachycheles monilifer

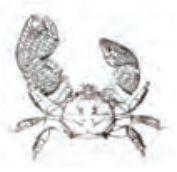

6

2

10

\section{Petrolisthes galathinus}

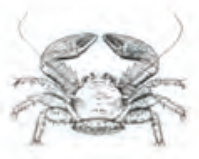

12

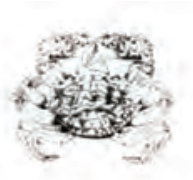

1

6 Pilumnus reticulatus

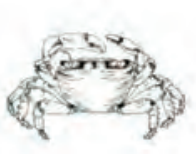

8 Petrolisthes armatus

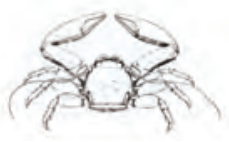

1

1

8

7 Pachygrapsus transversus

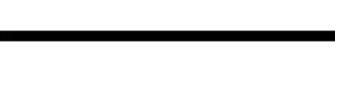

1
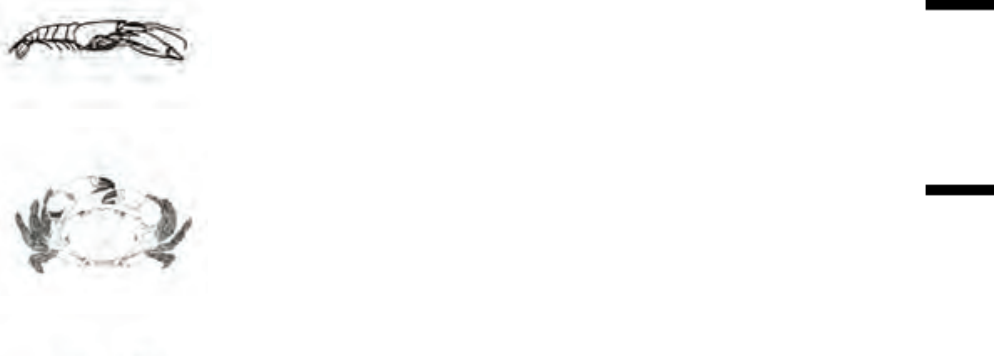

1

8

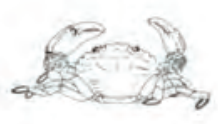


Tabela 3. Continuação...

\begin{tabular}{|c|c|c|c|c|c|c|c|}
\hline \multirow{2}{*}{\multicolumn{2}{|c|}{ Espécies }} & Meses & 1 & 3 & 5 & 7 & 9 \\
\hline & & Data & $18 / 10 / 06$ & $12 / 12 / 06$ & $21 / 02 / 07$ & $06 / 04 / 07$ & $14 / 06 / 07$ \\
\hline 12 Callinectes danae & & & 1 & & & & \\
\hline
\end{tabular}

13 Pleoticus muelleri
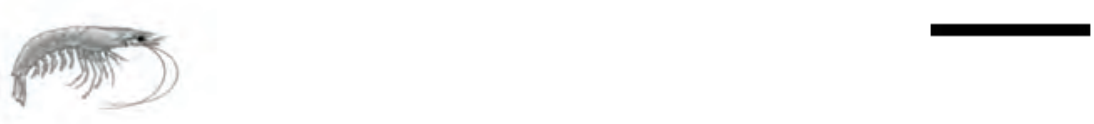

14 Libinia spinosa

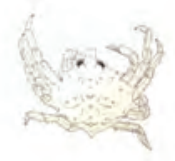

1

15 Porcellana platycheles
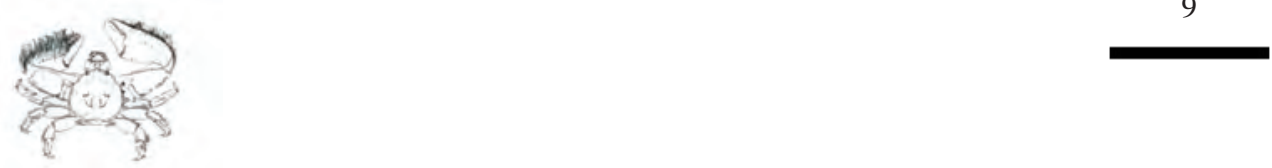

16 Panopeus lacustris

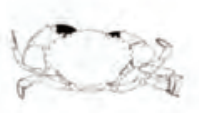

17 Panopeus rugosus
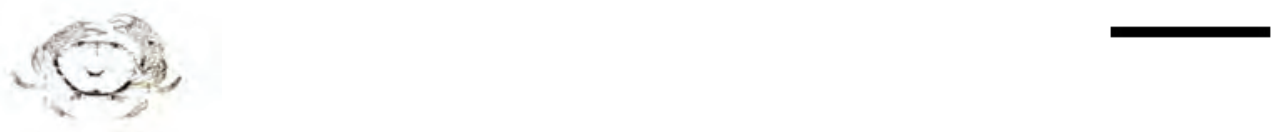

18 Panopeus austrobesus
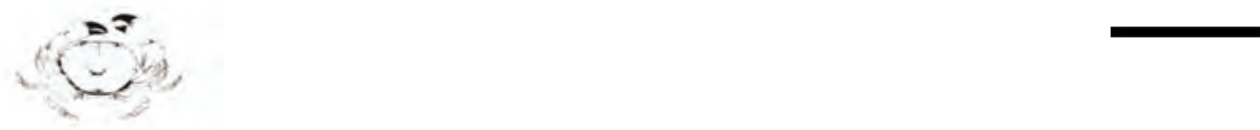

19 Porcellana sayana
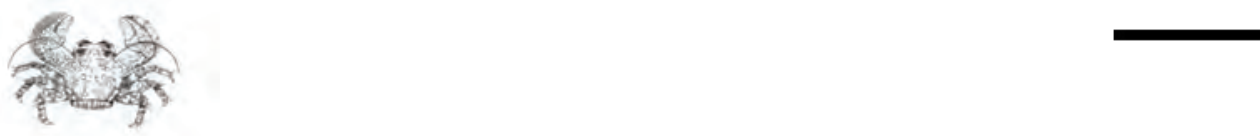

20 Pilumnoides coelhoi
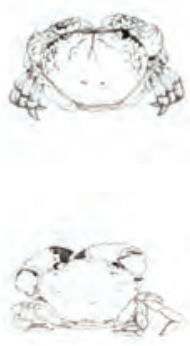
estando dependente da variação da temperatura (Ferreira et al. 2006). A eliminação de gametas (desova) promove perdas significativas no peso dos mexilhões (Marenzi \& Branco 2006). Esses fatores podem explicar a redução na velocidade de crescimento e diminuição do peso que ocorreu do terceiro para o quinto mês neste estudo. Além destes fatores, um causador de grande estresse para esses organismos é a predação por platelmintos, gastrópodes, equinodermos, peixes e aves que ocorre em maior intensidade nos meses mais quentes (Freitas 1997, Cochôa \& Magalhães 2008).

A produtividade média por metro de corda de cultivo é de $13 \mathrm{~kg}$ de mexilhões, e a fauna associada pode representar até $30 \%$ do peso total das cordas (Marenzi \& Branco 2006). Em contraste, no presente estudo, os crustáceos representaram apenas $0,62 \%$ (38,77 g no mês de maior peso). Nenhuma espécie apresentou grande contribuição em peso, embora a maioria tenha sido abundante numericamente. Os epibiontes embora constituam os competidores mais sérios dos mexilhões (Marques \& Pereira 1989) na Enseada da Armação do Itapocoroy, os crustáceos decápodos não constituem ameaça no que concerne ao peso como mencionado em outros cultivos (Choncheunchob 1980, Mattsson \& Lindén 1983). O elevado peso da fauna associada registrada pelos referidos autores se deve à presença de cirripédios, poliquetos tubícolas e outros animais coloniais que contribuem de maneira significativa para o peso total das cordas.

A biomassa da fauna de decápodos associados acompanhou o crescimento dos mexilhões até o sétimo mês, quando o seu valor atingiu o máximo; após, houve um significativo decréscimo juntamente com a sua abundância. Os epibiontes são competidores mais sérios dos mexilhões (Marques \& Pereira 1989). Cirripédios e ascídias sobre as valvas dos mexilhões dificultam o crescimento e, consequentemente, afetam a fauna associada (Marenzi \& Branco 2006). Estes observaram que a partir de $70 \mathrm{~mm}$ de altura de concha, os mexilhões tendem a reduzir o seu crescimento, certamente pela diminuição da eficiência na limpeza da concha com o pé (Loo \& Rosembreg 1983), facilitando a fixação de epibiontes (Andreu 1976). Embora os mexilhões deste estudo tenham atingido uma altura de concha próxima de $70 \mathrm{~mm}$ apenas no nono mês (junho), a súbita redução dos decápodos em abundância e biomassa, provavelmente se deu devido aos fortes ventos de leste que normalmente atingem diretamente a Armação do Itapocoroy nos meses de inverno e primavera (Marenzi \& Branco 2006), um fato largamente conhecido em outros cultivos (Choncheunchob 1980, Mattsson \& Lindén 1983), e que provavelmente desalojaram os mexilhões ou tornaram as cordas locais não estáveis para os decápodos habitarem. Desta forma, o tamanho dos mexilhões teve influência sobre a fauna de decápodos associada, porém as condições adversas de tempo que ocorreram durante esse mês de amostragem também tiveram importância na alteração do número de organismos.

Os estudos sobre fauna e flora associadas às cordas de cultivo de mexilhões realizada no Estado de Santa Catarina trataram parte considerável dos crustáceos decápodos ao nível de família ou superfamília (Marenzi \& Branco 2006), tornando difícil uma comparação com o presente estudo. Um total de 38 espécies de crustáceos associadas às cordas de mexilhões foi registrado neste Estado, além daquelas não identificadas ao nível específico (Freitas 1997, Marenzi \& Branco 2006, Leite 2007 e o presente estudo). Destas apenas três (Freitas 1997 e Marenzi \& Branco 2006), e duas (Leite 2007) espécies de decápodos foram registradas neste biótopo. Marenzi \& Branco (2006) encontraram ainda representantes da família Xanthidae MacLeay, 1838 (sensu lato). Certamente, esta baixa riqueza de espécies comparativamente ao presente estudo (21 espécies) está relacionada com a identificação ao nível taxonômico mais elevado nos trabalhos anteriores. Deste litoral, as seguintes cinco espécies foram comuns com o presente estudo:
Pachygrapsus transversus, Callinectes danae, Menippe nodifrons, Petrolisthes armatus, P. galathinus, perfazendo menos de $25 \%$ de similaridade de espécies. Outras espécies identificadas ao nível de gênero pelos referidos autores como Hexapanopeus sp. e Petrolisthes sp. poderiam também serem comuns com o presente estudo.

No estudo restrito aos decápodos braquiúros no litoral norte do Estado de São Paulo, Camargo (2011) encontrou 16 espécies, ou seja, quatro a mais do que no presente estudo (12 espécies de braquiúros). Sete espécies foram comuns no litoral do dois estados: Libinia spinosa, Hexapanopeus paulensis, Acantholobulus schmitti, Menippe nodifrons, Panopeus austrobesus, Pilumnus reticulatus e Pachygrapsus transversus, perfazendo $50 \%$ de similaridade de espécies de braquiúros. A espécie mais abundante naquele Estado foi Panopeus austrobesus com 711 indivíduos, ao passo que no presente estudo foi Pilumnus dasypodus com 244 indivíduos (total registrado em nove meses de cultivo). Estas diferenças na riqueza, composição e abundância das espécies em biótopos similares de litorais com clima distintos ressaltam a importância das variáveis abióticas reinantes nos respectivos ecossistemas.

Ricklefs (2003) afirma que substratos mais homogêneos abrigam uma riqueza de espécies menor do que aquelas verificadas para substratos mais heterogêneos. Neste contexto, as cordas de cultivo de mexilhões podem ser consideradas os substratos mais heterogêneos conhecidos. Corroboram esta hipótese as riquezas de espécies de decápodos braquiúros registradas em outros substratos biológicos: 14 espécies sobre as colônias do briozoário Schizoporella unicornis (Johnston, 1847); (Mantelato \& Souza-Carey 1998) e 12 espécies sobre as comunidades fitobentônicas de um costão rochoso (Széchy et al. 2001).

As espécies encontradas nesse estudo pertencem a nove famílias, sendo Porcellanidae a mais rica e abundante, apresentando sete espécies. Esta família, apesar de pouco citada em outras pesquisas, pode ter sua elevada abundância nas cordas de mexilhões explicada pela plasticidade adaptativa de suas 230 espécies que habitam uma grande variedade de habitats, entre-marés e sublitorais, como bancos algas, corais, esponjas, fissuras das rochas, embaixo de pedras, incluindo os bancos naturais de mexilhões (Rodriguez 1980). Além disso, apesar destes animais serem primariamente filtradores, podem manipular pedaços de alimento com os quelípodos e aproveitar detritos depositados no substrato (Kropp 1981), podendo ainda ocorrer como comensais de algumas espécies de fundos duros (anêmonas-do-mar) e moles (estrelas-do-mar) (Silva et al. 1989). Os porcelanídeos Pachycheles laevidactylus e Petrolisthes armatus são encontrados em bancos naturais de mexilhões; Porcellana sayana em fundos de lama, substrato característico da região de Enseada da Armação do Itapocoroy e sobre conchas e como comensais de paguros e gastrópodes; Pachycheles monilifer, Porcellana platycheles e Pisidia brasiliensis são mais comuns em substratos rochosos de ambientes naturais (Melo 1999).

Marenzi \& Branco (2006) na Enseada da Armação do Itapocoroy, SC registraram maior abundância de representantes da antiga família Xanthidae MacLeay, 1838 nas cordas de mexilhões. Estes dados coincidem com o presente estudo, pois nove espécies desta superfamília totalizaram 304 indivíduos, perfazendo 60,67\% do total. Além de serem importantes numericamente, muitos Xanthidaes são predadores bentônicos, mas frequentemente desprezados devido ao seu pequeno tamanho e comportamento críptico.

Silliman et al. (2004) sugeriram que estes caranguejos quebradores de conchas atuam como forças importantes na estruturação das comunidades. Entretanto, com exceção de Menippe nodifrons, os representantes desta supefamília do presente estudo foram de pequeno a médio porte (Marochi \& Masunari 2011) e, portanto, 
quebras de conchas de mexilhões por estes caranguejos não seriam possíveis. Mesmo os Menippe nodifrons potencialmente predadores de moluscos registrados no presente estudo foram juvenis de pequeno porte e não atingiram tamanhos maiores do que $2 \mathrm{~cm}$ de largura de carapaça (os adultos desta espécie podem atingir até $78,82 \mathrm{~cm}$ (Marochi \& Masunari 2011), sendo portanto incapazes de realizar tal atividade. Como a colonização por estes caranguejos aconteceu apenas no sétimo mês (com aumento significativo de abundância no último mês, quando os mexilhões já haviam atingido cerca de $6 \mathrm{~cm}$ de altura de concha, é muito provável que estes animais estariam predando exclusivamente os componentes da fauna associada de porte compatível com o tamanho dos mesmos. No início do cultivo, quando os mexilhões tinham apenas $25 \mathrm{~mm}$ de concha, e portanto passíveis de serem predados, estes caranguejos estavam ausentes. Com o mesmo raciocínio, as demais espécies de decápodos utilizaram as cordas de cultivo dos mexilhões como um substrato alternativo de moradia e fonte de alimento diferente da carne do mexilhão adulto. Entretanto, como a desova dos mexilhões Perna perna em Santa Catarina ocorre principalmente nos meses de abril e maio, e estes juvenis encontram local adequado para seu desenvolvimento nas próprias cordas de mexilhões (Cochôa \& Magalhães 2008), este pode ser mais um dos fatores do aparecimento de Menippe nodifrons, potenciais predadores no sétimo e nono mês de amostragem (abril e junho), em busca dos mexilhões juvenis que nasceram das referidas desovas e se fixaram aos mexilhões adultos.

Segundo Tenore \& Gonzales (1975), o abrigo disponibilizado pelas cordas de mexilhões é um dos principais fatores que regulam o estabelecimento de crustáceos nos cultivos de mexilhões em Ría de Arosa, Espanha. Por outro lado, Mantelatto \& Souza-Carey (1998) concluíram que o abrigo para caranguejos braquiúros disponibilizado pelo briozoário Schizoporella unicornis (Johnston, 1847) foi o principal recurso que atraiu estes organismos; recurso este que foi incrementado à medida que o substrato biológico foi crescendo em tamanho e complexidade. Além disso, Széchy et al. (2001) ressaltaram que as espécies associadas aos mexilhões apresentam dimensões compatíveis ao espaço oferecido como abrigo entre as conchas: estas são de pequeno porte durante as fases iniciais do desenvolvimento juvenil do substrato, o que demonstra a importância do abrigo como recurso para estes animais. O registro de uma relação diretamente proporcional entre a abundância de caranguejos e o crescimento do substrato dos mexilhões do presente estudo permite inferir que ambos os fatores devem estar atuando nas cordas de cultivo. Com o crescimento das conchas dos mexilhões, houve um incremento de espaços entre as mesmas e, portanto, maior oferta de abrigos para a colonização dentre as conchas de maiores dimensões.

Não houve relação entre o número e o peso das espécies mais abundantes. Em uma corda pode haver espécies abundantes, mas com pouca biomassa e outras raras, mas com muita biomassa, contribuindo de maneira semelhante para o peso final da corda de mexilhões. Destaca-se como exemplo Menippe nodifrons, que apresentou poucos indivíduos, porém com maior biomassa. Por outro lado, a espécie com maior biomassa total foi Pilumnus dasypodus, cujos indivíduos são de muito menor porte do que os de Menippe nodifrons, porém numericamente superior (total de 183 indivíduos somente no mês sete). Assim, a espécie que apresentou maior biomassa foi Menippe nodifrons e a espécie mais abundante e frequente foi Pilumnus dasypodus.

Não se conhecem estudos que tratem especificamente da sucessão de crustáceos decápodos associados às cordas de mexilhões, mas pode-se afirmar que houve substituição e acréscimo de espécies no decorrer do tempo do cultivo. Entretanto, espécies que foram registradas no $1^{\circ}$ mês- Callinectes danae, Pachygrapsus transversus $\mathrm{e}$ Petrolisthes armatus devem ser consideradas ocasionais mais do que pioneiras, em função das frequências muito baixas. Foi observado que a rede de algodão ainda estava presente no primeiro mês em duas das três cordas amostradas, onde não foi registrado qualquer crustáceo decápodo. A terceira corda apresentou as três espécies citadas, com um representante cada uma. A malha de algodão pode ter interferido na entrada e colonização dos braquiúros. Outro fator para a baixa riqueza de espécies no primeiro mês de amostragem foi o tamanho abaixo do ideal das sementes, que propiciou o aumento da densidade e, portanto, pouco espaço disponível para os organismos associados.

De uma forma geral, o aumento na abundância e na diversidade de espécies com o passar do tempo indica que a comunidade se tornou mais complexa em termos de arquitetura e de composição trófica. A diversidade máxima registrada no último mês $(1,31)$ pode indicar uma tendência ao aumento deste índice em direção à estabilidade da assembleia. A colonização da espécie mais abundante, Pilumnus dasypodus, teve início somente após três meses do início do cultivo. A queda acentuada na abundância desta espécie no nono mês pode indicar fuga para outros habitats, pois, a temperatura da água tende a cair significativamente em junho (nono mês). Outras espécies como $P$. reticulatus, $P$. laevidactylus, $P$. galathinus, $P$. brasiliensis e $S$. fritzmuelleri mostraram indícios de colonização e estabelecimento das populações. $M$. nodifrons também pertenceria a esta categoria, porém, como esta espécie foi representada exclusivamente por juvenis, uma migração para outras áreas naturais deve certamente ter ocorrido. Por outro lado, espécies que surgiram tardiamente (somente no último mês) como todos os Panopeus, H. paulensis, Porcellana sayana e $P$. platycheles indica que as mesmas necessitam de habitats mais complexos para iniciar a sua colonização.

Algumas espécies tiveram uma ocorrência rara neste trabalho, como Callinectes danae, o único siri dessa amostragem, e animal típico de substratos não-consolidados (Calado \& Souza 2003) de hábito predador e oportunista (Leite 2007), porém sua presença em cordas de cultivo de mexilhões já foi registrada anteriormente por e Freitas (1997). Sua captura deve ter sido acidental, pelo fato de possuir capacidade de natação e de explorar a coluna d'água. Entretanto, a julgar pela dieta constituída de moluscos, poliquetos e crustáceos (Branco \& Verani 1997), a possibilidade destes siris explorarem a fauna associada às cordas em busca de alimento não pode ser descartada. Juntamente com $P$. transversus e $P$. armatus, este siri constitui um grupo de braquiúros que visitou as cordas no início do cultivo, mas não estabeleu populações nas mesmas. Outras espécies raras, como L. spinosa. A. schimitti e P. muelleri, certamente exploram as cordas de cultivo, mas não conseguem se estabelecer como populações.

O predomínio dos moluscos na dieta dos braquiúros foi registrado por vários autores: Ropes (1968), Laughlin (1982), Wear \& Haddon (1987) e Haefner (1990). Elner (1978) e Seed (1980) relatam a versatilidade dos braquiúros em capturar suas presas que vivem associadas aos mexilhões. Elner \& Lavoie (1983) verificam o mesmo nos cultivos de ostras, Stevens et al. (1982) de outros moluscos e Kneib (1982) de peixes.

Os camarões da família Alpheidae são comuns e ocupam cavidades naturais em diferentes substratos, por isso também podem ser encontrados em cordas de mexilhões (Calado \& Souza 2003). As três espécies registradas de Panopeus são bem diferentes entre si em relação ao hábito alimentar e ao habitat: $P$. austrobesus e $P$. rugosus vivem em substrato não-consolidado (Melo 1996), enquanto, P. lacustris, em costões e outros locais de substrato consolidado. Essas espécies podem ser observadas junto às ostras (Melo 1996) e, segundo Calado \& Souza (2003), possuem importância na teia trófica de vertebrados. Sua ocorrência nos meses finais de amostragem pode ser explicada pela maior complexidade de habitat com disponibilidade suficiente de alimento e abrigo para fuga dos vertebrados. Do 
litoral de Santa Catarina são conhecidas 38 espécies de crustáceos decápodos associados aos cultivos de mexilhões, das quais 21 foram registradas para as cordas de mexilhões do presente estudo, indicando que este biótopo contribui significativamente para o incremento da biodiversidade carcinológica. Certamente, maiores esforços amostrais poderão elevar o valor em relação ao obtido, pois, espécies raras podem estar subestimadas devido às suas baixas abundâncias.

\section{Agradecimentos}

À equipe do CEMar: Centro Experimental de Maricultura - Penha da Universidade do Vale do Itajaí - UNIVALI pelo apoio logístico; aos Oceanógrafos Gil Reiser e Felippe Daros pelo auxílio em campo; ao Biólogo Murilo Z. Marochi pela confirmação da identificação dos crustáceos braquiúros desse estudo; Ao Prof. Dr. Henry Louis Spach da Universidade Federal do Paraná pela disponibilização do programa estatístico. Esta é a contribuição No 1863 do Departamento de Zoologia UFPR.

\section{Referências Bibliográficas}

ANDREU, B. 1976. El cultivo del mejillon en Europa. An. Acad. Bras. Cienc. 45(Supl.):1-32.

BRANCO, J.O. \& VERANI, J.R. 1997. Dinâmica da alimentação natural de Callinectes danae Smith, (Decapoda, Portunidae) na Lagoa da Conceição, Florianópolis, Santa Catarina, Brasil. Rev. Bras. Zool. 14(4):1003-1018.

CALADO, T.C.S. \& SOUSA, E.C. 2003. Crustáceos do complexo estuarinolagunar Mundaú/Manguaba Alagoas. Fundação de Amparo a Pesquisa de Alagoas - FAPEAL, Maceió.

CAMARGO, F.V. 2011. Biodiversidade de caranguejos braquiúros (Crustacea: Decapoda) associados ao cultivo comercial de mexilhão Perna perna (Linnaeus, 1758) (Molusca, Bivalvia) no Litoral Norte do Estado de São Paulo. Dissertação de mestrado, Universidade Estadual Paulista, Botucatu.

CARVALHO, J.L.B., SCHETTINI, C.A.F. \& TRUCCOLO, E.C. 1999. Aspectos hidrodinâmicos da Enseada da Armação do Itapocoróy, SC. Notas téc. FACIMAR 3:99-109.

CHONCHEUNCHOB, P. 1980. Handing culture of the green mussel (Mytilus smarangdinus (Chemnatz) in Thailand. Editora ICLARM, Manila.

COCHÔA, A.R. \& MAGAlHÃES, A.R.M. 2008. Perdas de sementes de mexilhão Perna perna (L., 1758), cultivado na Baía Norte - Ilha de Santa Catarina/SC. Bol. Inst. Pesca 34(1):1-10.

CONNELL, J.H. \& SLATYER, R.O. 1977. Mechanisms of succession in natural communities and their role in community stability and organization. Am. Nat. 111:1119-1144.

DeGRAAF, J.D. \& TYRRELL, M.C. 2004. Comparison of the feeding rates of two introduced crab species, Carcinus maenas and Hemigrapsus sanguineus, on the blue mussel, Mytilus edulis. Northeast. Nat. 11(2):163-166.

DE GRAVE, S., PENTCHEFF, N.D., AHYONG, S.T., CHAN, T-Y., CRANDALL, K.A., DWORSCHAK, P.C., FELDER, D.L., FELDMANN, R.M., FRANSEN, C.H.J.M., GOULDING, L.Y.D., LEMAITRE, R., LOW, M.E.Y., MARTIN, J.W., NG, P.K.L., SCHWEITZER, C.E., TAN, S.H., TSHUDY, D. \& WETZER, R. 2009. A classification of living and fossil genera of decapod crustaceans. Raffles Bull. of Zoo. Supplement 21:1-109.

ELNER, R.W. 1978. The mechanics of predation by the shore crab, Carcinus maenas (L.), on the edible mussel, Mytilus edulis L. Oecologia. 36(3):333-344.

ELNER, R.W. \& LAVOIE, R.E. 1983. Predation on American oysters (Crassostrea virginica (Gmelin)) by American lobsters (Homarus americanus Say), and mud crabs (Neopanopesayi (Smith)). J. Shellfish Res. 3(2):129-134.

FERREIRA, J.F \& MAGALHÃES, A.R.M. 2004. Cultivo de mexilhões. In Aqüicultura - experiências brasileiras (C.R Poli, A.T.B. Poli, E. Andreatta, $\&$ E. Beltrame, eds.). Multitarefa, Florianópolis.
FERREIRA, J.F., OLIVEIRA-NETO, F.M., MARENZZI, A.C., TUREK, C. \& SILVA, R.T. 2006. Coletores de sementes de mexilhões - a opção do mitilicultor catarinense para retomar o crescimento da produção. Panor. Aquic. 43-48.

FREITAS, M. 1997. Incrustações biológicas no mexilhão Perna perna (Mollusca, Bivalvia), cultivado na Ilha de Ratones, SC: efeito da exposição ao ar. Dissertação de mestrado, Universidade Federal de Santa Catarina, Florianópolis.

GILLER, P.S. 1984. Community structure and niche. Chapman \& Hall, London. http://dx.doi.org/10.1007/978-94-009-5558-5_2

HAEFNER, P.A.J.R. 1990. Natural diet of Callinectes ornatus (Brachyura: Portunidae) in Bermuda. J. Crustacean Biol. 10(2):236-246.

HAMILTON, D.J. \& NUDDS, T.D. 2003. Effects of predation by common eiders (Somateria mollissima) in an intertidal rockweed bed relative to an adjacent mussel bed. Mar. Biol. 142(1):1-12.

HENRIQUES, M.B., MARQUES, H.L.A., PEREIRA, O.M. \& BASTOS, G.C. 2004. Aspectos da estrutura populacional do mexilhão Perna perna, relacionados à extração em bancos naturais da baía de santos, Estado de São Paulo, Brasil. Bol. Inst. Pesca 30(2):117-126.

INGLIS, G.J. \& GUST, N. 2003. Potential indirect effects of shellfish culture on the reproductive success of benthic predators. J. Appl. Ecol. 40(6):1077-1089.

KAUTSKY, N. \& FOLKE, C. 1991. Ecological economic principles for aquaculture development. In Nutritional Strategies and Aquaculture Waste (C.B. Cowey \& C.Y. Cho, eds.). University of Guelph, Ontario.

KNEIB, R.T. 1982. The effects of predation by wading birds (Ardeidae) and blue crab (Callinectes sapidus) on the population size structure of the common mummichog, Fundulus heteroclitus. Estuar. Coast. Shelf Sci. 14:159-163. http://dx.doi.org/10.1016/S0302-3524(82)80042-X

KROPP, R.K. 1981. Additional porcdain crah feeding methods (Decapoda, Porcelanidae). Crustaceana 40(3):307-310. http://dx.doi. org/10.1163/156854081X00778

LAPPALAINEN, A., WESTERBOM, M. \& VESALA, S. 2004. Blue mussels (Mytilus edulis) in the diet of roach (Rutilus rutilus) in outer archipelago areas of the western Gulf of Finland, Baltic Sea. Hidrobiologia. 514(1-3):87-92. http://dx.doi.org/10.1023/ B:hydr.0000018209.10032.0c

LAUGHLIN, R.A. 1982. Feeding habits of the blue crab, Callinectes sapidus Rathbun, in the Apalachicola estuary, Florida. Bull. Mar. Sci. 32:807-822.

LEITE, L.A. 2007. Influência da predação, parasitismo e densidade de sementes nas perdas de mexilhões Perna perna (L., 1758) cultivados na Baía Norte da Ilha de Santa Catarina. Dissertação de mestrado, Universidade Federal de Santa Catarina, Florianópolis.

LOO, L.O. \& ROSENBERG, R. 1983. Mytilus edulis culture: growth and production in Western Sweden. Aquaculture. 35:137-150.

MANTELATTO, F.L.M. \& SOUZA-CAREY, M.M. 1998. Brachyura (Crustacea, Decapoda) associated to Schizoporella unicornis (Bryozoa, Gymnolaemata) in Ubatuba Bay (SP), Brazil. Braz. Arch. Biol. Techn. 41(2):212-217.

MANZONI, G.C. \& LACAVA, L.A. 1998. Crescimento doa gastrópodes Thais (Stramonita) haemastoma e Cymatium parthenopeum parthenopeum em cultivo experimental na Enseada da Armação do Itapocoróy (26ํำ'S - 48³6'W)(Penha - SC). Notas téc. FACIMAR 2: 167-173.

MARENZI, A.W.C. 1999. O uso de mecanização nos cultivos de mexilhões Perna perna em Santa Catarina. Agrárias 18(1-2):155-163.

MARENZI, A.W.C. \& BRANCO, J.O. 2006. O cultivo do mexilhão Perna perna no Município de Penha - SC. In Bases ecológicas para um desenvolvimento sustentável: estudos de caso em Penha - SC. (J.O. Branco \& A.W.C. Marenzi, eds.). Editora da UNIVALI, Itajaí, p.227-244.

MARENZI, A.W.C. \& MANZONI, G.C.M. 1998. Cultivo de moluscos marinhos: estabelecimento de novo ecossistema em regiões costeiras de Santa Catarina. In IV Simpósio de Ecossistemas Brasileiros. Águas de Lindóia, v.3, p.438- 442. 
MAROCHI, M.Z. \& MASUNARI, S. 2011. Os caranguejos Eriphiidae, Menippidae, Panopeidae e Pilumnidae (Crustacea Brachyura) de águas rasas do litoral do Paraná, com chave pictórica de identificação para as espécies. Biota Neotrop. 11(3): http://www.biotaneotropica.org.br/ v11n3/pt/abstract?inventory+bn00211032011 http://dx.doi.org/10.1590/ S1676-06032011000300001

MARQUES, H.L.A. 1998. Criação comercial de mexilhões. Editora Nobel, São Paulo.

MARQUES, H.L.A. \& PEREIRA, R.T.L. 1989. Levantamento e dimensionamento preliminares das áreas mais favoráveis para a prática da mitilicultura no litoral do Município de Ubatuba, Estado de São Paulo $\left(23^{\circ} 26^{\prime} \mathrm{S}, 045^{\circ} 04^{\prime} \mathrm{W}\right)$. Bol. tec. - Inst. Pesca 13.

MATTSSON, J.C. \& LINDÉN, O. 1983. Benthic macrofauna succession under mussels Mytilus edulis L. (Bivalvia) cultured on hanging longlines. Sarsia 68:97-102.

MELO, G.A.S. 1996. Manual de identificação dos Brachyura (caranguejos e siris) do litoral brasileiro. Plêiade/FAPESP, São Paulo.

MELO, G.A.S. 1999. Manual de identificação dos Crustacea Decapoda do litoral brasileiro: Anomura, Thalassinidea, Palinuridea, Atacidea. Plêiade; FAPESP, São Paulo.

NG, P.K.L., GUINOT, D. \& DAVIE, P.J.F. 2008. Systema Brachyurorum: part I. An annotated checklist of extant brachyuran crabs of the world. Raffles Bull. Zool. 17:1-286.

RICKLEFS, R.E. 2003. Economia da Natureza. 6. ed. Editora Guanabara Koogan, São Paulo.

RODRIGUEZ, G. 1980. Los crustaceos decapodos de Venezuela. Instituto Venezodano de Investigaciones Cientíticas, Caracas, 494p.

ROPES, J.W. 1968. The feeding habits of the green crab, Carcinus maenas (L.). Fishery Bull. Fish Wildl. Serv. U.S. 67(2):183-203.

SANTOS, W.A. 2006. Importância econômica dos costões rochosos. Saúde \& Amb. Rev. 1(2):51-59.

SCHETTINI, C.A.F., CARVALHO, J.L.B. \& TRUCCOLO, E.C. 1999 Aspectos hidrodinâmicos da enseada da Armação do Itapocoroy, SC. Notas téc. FACIMAR 3:99-109.

SCHETTINI, C.A.F., RESGALLA JUNIOR, C. \& KUROSHIMA, K.N. 1997. Avaliação preliminar da taxa sedimentar na região de cultivo de moluscos (Perna perna) na enseada da Armação-SC. Notas téc. FACIMAR 1: 1-8.

SEED, R. 1976. Ecology. In Marine Mussels: their ecology and physiology. (B.L. Bayne, ed). Cambridge University Press, Cambridge.
SEED, R. 1980. Predatior-prey relationships between the mud crab Panopeus herbstii, the blue crab, Callinectes sapidus and the Atlantic ribbed mussel Geukensia (=Modiolus) demissa. Estuar. Coast. Mar. Sci. 11:445-448. http://dx.doi.org/10.1016/S0302-3524(80)80067-3

SILLIMAN, B.R., LAYMAN, C.A., GEYER, K. \& ZIEMAN, J.C. 2004. Predation by the black-clawed mud crab, Panopeus herbstii, in MidAtlantic salt marshes: further evidence for top-down control of marsh grass production. Estuaries. 27(2):188-196.

SILVA, B.M.G., BRAGA, A.C. \& D'INCAO, F. 1989. Porcellanidae (Decapoda Anomura) de Santa Catarina e Rio Grande do Sul, Brasil. Iheringia, Série Zool. 69:131-146.

STEVENS, B.G., ARMSTRONG, D.A. \& CUSIMANO, R. 1982. Feeding habits of the dungeness crab Cancer magister as determined by the index of relative importance. Mar. Biol. 72:135-145.

SUPLICY, F.M. 2000. Capacidade de suporte nos cultivos de moluscos. Panor. Aquic. 10(57):21-4.

SZÉCHY, M.T.M., VELOSO, V.G. \& PAULA, E.J. 2001. Brachyura (Decapoda Crustacea) of phytobenthic communities of the sublittoral region of rocky shores of Rio de Janeiro and São Paulo, Brazil. J. Trop. Ecol. 42(2):231-242.

TENORE K.R. \& GONZALES, N. 1975. Food chain patterns in the Ría de Arosa, Spain; an area of intense mussel aquaculture. In European Symposium on Marine Biology (G. Persoone \& E. Jaspers, eds.). Universa Press, Wetteren, v.2, p.601-619.

TISDELL, C. 1991. Development of aquaculture and the environment: coastal conflicts, and giant clam farming as a case. Int. J. Environ. Stud. 39:35-44. http://dx.doi.org/10.1080/00207239108710679

TSUTSUMI, H., KIKUCHI, T., TANAKA, M., HIGASHI, T., IMASAKA, K. \& MYASAKI, M. 1991. Benthic faunal sucession in a cove organically polluted by fish farming. Mar. Pollut. Bull. 23:233-238.

WATERSTRAT, P., CHEW, K., JOHNSON, K. \& BATTIE, J.H. 1980. Mussel culture: a West Coast perspective. In Mussel culture and harvest: a north american perspective (R.A. Lutz, \& I.C.D. Center, eds.). New York, v.1, p.141-163.

WEAR, R.G. \& HADDON, M. 1987. Natural diet of the crab Ovalipes catharus (Crustacea, Portunidae) around central and northen New Zealand. Mar. Ecol. Prog. Ser. 35:39-49.

WITMAN, J.D. \& SUCHANEK, T.H. 1984. Mussels in flow: drag and dislodgment by epibionts. Mar. Ecol. Prog. Ser. 16:259-262.

YOUNG, J.A., BRUGERE, C. \& MUIR, J.F. 1999. Green grow the fishies$\mathrm{Oh}$ ? Environmental attributes in marketing aquaculture products. Aquac. Economics Manag. 3(1):7-17. 